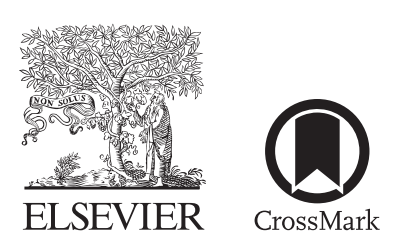

\title{
Vascular Trauma in Children-Review from a Major Paediatric Center
}

\author{
Sofia Morão, ${ }^{1}$ Rita S. Ferreira, ${ }^{2}$ Nelson Camacho, ${ }^{2}$ Vanda Pratas Vital, ${ }^{1}$ João Pascoal, ${ }^{1}$ \\ M. Emilia Ferreira, ${ }^{2}$ L. Mota Capitão, ${ }^{2}$ and Frederico B. Gonçalves, ${ }^{2,3}$ Lisbon, Portugal
}

\begin{abstract}
Background: Traumatic noniatrogenic vascular injuries in children are rare and rarely discussed in literature. Pediatric vascular injuries pose a set of challenges mainly because of continued growth and development in a child or adolescent. The purpose of the study is to characterize management strategies and outcomes in these cases.

Methods: This is a single-center retrospective review of patients less than age 18 years (pediatric age) with acute, noniatrogenic traumatic vascular injuries between January 2009 and December 2015. Patient's demographics, injury characteristics, surgical management, complications, and follow-up were analyzed.

Results: From 2009 to 2015, 3277 children with traumatic injuries were treated, of which 21 $(0.6 \%)$ had 23 significant vascular injuries: 17 arterial and 6 venous injuries. The majority were males $(n=16)$, and the median age was 14 years (range 1 to 16 years). Penetrating injuries were the predominant mechanism $(n=21)$, mainly by glass $(n=13)$. At presentation, 4 patients were hemodynamically unstable, 3 of them in hypovolemic shock. All patients were managed operatively. Operations for arterial injuries included 5 primary arterial repairs, 4 repairs using vein grafts and 8 ligations. The following adjunct procedures were necessary: one 4-compartment leg fasciotomy due to associated soft tissue trauma, 8 tendon repairs, and 11 nerve repairs. Operations for venous injuries included 4 ligations and 2 primary repairs. There were no intraoperative or postoperative deaths, major complications, or limb loss. The median length of stay in the hospital was 6 days (range: 2-23 days). The median time of follow-up was 52 months (range: 20-94 months). Ten patients did not have any sequelae, and 11 patients reported impaired mobility and/or decreased sensation, which was transitory in most cases and related to associated neurological or muscle tendon injuries. All reconstructions remained patent over the course of follow-up. No limb asymmetry was observed.

Conclusions: Noniatrogenic pediatric vascular trauma is uncommon. Penetrating mechanism is more common than blunt and extremities are more frequently affected. Overall complications come from associated injuries to tendons and nerves.
\end{abstract}

Conflict of Interest: The authors have no conflicts of interest to disclose.

${ }^{I}$ Department of Paediatric Surgery, Hospital Dona Estefânia, Centro Hospitalar Lisboa Central, Lisbon, Portugal.

${ }^{2}$ Department of Angiology and Vascular Surgery, Hospital Santa Marta, Centro Hospitalar Lisboa Central, Lisbon, Portugal.

${ }^{3}$ NOVA Medical School, Lisbon, Portugal.

Correspondence to: Sofia Morão, Department of Paediatric Surgery, Hospital Dona Estefânia, Centro Hospitalar Lisboa Central, Rua Jacinta Marto 1169-045, Lisboa,Portugal; E-mail:sophia.morao@gmail.com

Ann Vasc Surg 2018; 49: 229-233

https://doi.org/10.1016/j.avsg.2017.10.036

(C) 2018 Elsevier Inc. All rights reserved.

Manuscript received: September 11, 2017; manuscript accepted: October 24, 2017; published online: 9 February 2018

\section{BACKGROUND}

Vascular injuries in pediatric patients are rare. Vascular injury has been well studied in the adult population with a significant amount of data from the management military casualties translated to civilian practice. Regarding the pediatric population, however, sparse reports have dealt with this topic. Most of them include iatrogenic etiologies from cardiac catheterization or percutaneous vascular access procedures, ${ }^{1,2}$ and just a few series exists describing only noniatrogenic vascular injuries. Pediatric vascular injuries have unique characteristics since 
children are more prone to vasospasm, have smaller diameter vessels, and have vessels that need to grow with the child. ${ }^{3,4}$ The combination of a technically difficult condition, with little established protocol and potentially devastating outcomes makes pediatric vascular trauma an important issue. The purpose of this study was to characterize management strategies and outcomes of traumatic vascular injuries at a tertiary pediatric center.

\section{PATIENTS AND METHODS}

The study follows the Helsinki declaration for research ethics. Informed consent was not necessary according to local guidelines and study's retrospective nature. It is a retrospective review of patients admitted to a single tertiary pediatric center with traumatic noniatrogenic vascular injuries between January 2009 and December 2015. The institution's registry was queried to identify patients less than 18 years of age (pediatric age limit considered in study's country) with an International Classification of Diseases, Ninth Revision code for a traumatic vascular injury (900.0-904.9). All data regarding demographic information, mechanism of injury, symptoms, hemodynamic status on presentation, identity of injured vessels, concomitant injuries, initial diagnostic procedures, operative therapy, approach, length of stay, complications, and follow-up were collected.

The study primary endpoints were early mortality, major morbidity, and vessel patency at followup. Additionally, we sought to determine if there were predictors of failure or consequences to the affected limb following vascular injury.

Discrete variables were presented as count and percentage, continuous variables as median and range. Statistical analysis was performed with SPSS 21.0.

\section{RESULTS}

During the study period, 3,277 patients with traumatic injuries were admitted, of which 21 $(0.64 \%)$ sustained 23 significant vascular injuries: 17 arterial and 6 venous injuries. Six patients were treated by vascular surgeons, 1 patient was treated by a plastic surgeon, and the other 14 patients by pediatric surgeons. Most patients were male $(n=16)$. The median age of the patients was 14 years (range: $1-16$ years). Eighteen were transferred from another hospital. The incidence of pediatric vascular trauma has been relatively stable over time (Fig. 1). All patients were healthy except one,

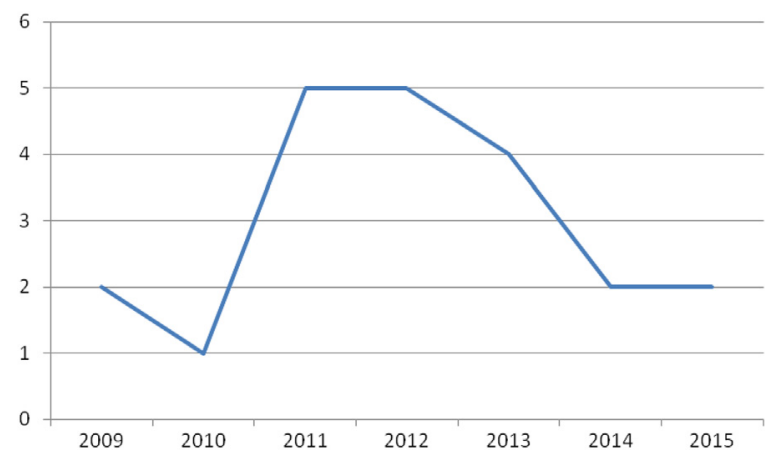

Fig. 1. Number of vascular injuries seen per year.

who had chronic renal disease. Penetrating injuries were the dominant mechanism of injury $(n=21)$, most commonly by glass $(n=13)$. One patient had a fracture associated with the arterial injury. Mechanism of injury is shown in Tables I and II by vessel injury. The most commonly injured vessels were the ulnar and radial arteries. Arterial injuries were all located in extremities, but only $4(24 \%)$ occurred in arteries of the lower limbs. Venous injuries were located in neck $(n=2)$, lower limbs $(n=3)$, and abdomen $(n=1)$.

At presentation, 4 patients were hemodynamically unstable (19\%) (11-year-old girl and 15-year-old boy both with lesion of femoral artery; 7-year-old boy with lesion of anterior tibial artery; and 11 year-old-boy with laceration of right suprahepatic vein) and 4 with acute limb ischemia (2 lesions of cubital artery, 1 lesion of anterior tibial artery, and 1 lesion of brachial artery). Eight patients were actively bleeding, 5 had a hematoma, and 1 had a fracture with neurovascular compromise. Two had an X-ray (search for foreign body and suspicion of fracture), 1 underwent Doppler ultrasound, 2 performed a computed tomography angiography ( 1 blunt abdominal trauma and 1 injury of the neck), and 1 had an esophagogram.

All patients in this series were managed operatively. The similar basic principle of proximal and distal control with vessel loops was applied in all cases before direct exploration of the affected vascular segment. Systemic heparinization was not used in any case, but arterial flushing with heparinized saline solution is generally applied.

Operations for arterial injuries included 5 end-toend anastomoses, 4 repairs using vein grafts interpositions (great saphenous vein was used in all cases), and 8 ligations. Whenever anastomoses were performed, interrupted sutures with monofilament nonabsorbable suture material were used. It was decided to reconstruct an injury to a single tibial vessel (posterior tibial artery) with vein graft 
Table I. Mechanism of arterial injury

\begin{tabular}{|c|c|c|c|c|c|c|c|c|c|}
\hline \multirow[b]{2}{*}{ Mechanism } & \multirow[b]{2}{*}{ Total } & \multicolumn{8}{|c|}{ Injured artery } \\
\hline & & Brachial & Radial & Ulnar & $\begin{array}{l}\text { Proper } \\
\text { palmar digital }\end{array}$ & $\begin{array}{l}\text { Common } \\
\text { palmar digital }\end{array}$ & Femoral & $\begin{array}{l}\text { Anterior } \\
\text { tibial }\end{array}$ & $\begin{array}{l}\text { Posterior } \\
\text { tibial }\end{array}$ \\
\hline Penetrating & 16 & & & & & & & & \\
\hline Glass & & & 2 & 7 & 1 & & 1 & 1 & \\
\hline Grass cutting machine & & & & & & & 1 & & \\
\hline Wood cutting machine & & & & & & 1 & & & 1 \\
\hline Box cutter & & & 1 & & & & & & \\
\hline Blunt & 1 & & & & & & & & \\
\hline Fall & & 1 & & & & & & & \\
\hline
\end{tabular}

Table II. Mechanism of venous injury

\begin{tabular}{|c|c|c|c|c|c|c|c|}
\hline \multirow[b]{2}{*}{ Mechanism } & \multirow[b]{2}{*}{ Total } & \multicolumn{6}{|c|}{ Injured vein } \\
\hline & & $\begin{array}{l}\text { External } \\
\text { Jugular }\end{array}$ & $\begin{array}{l}\text { Collaterals of } \\
\text { internal jugular }\end{array}$ & Femoral & Posterior tibial & Anterior tibial & Right suprahepatic \\
\hline Penetrating & 5 & & & & & & \\
\hline Glass & & & & & 1 & & \\
\hline Dog bite & & & 1 & & & & \\
\hline Toy & & 1 & & & & & \\
\hline Grass cutting machine & & & & 1 & & & \\
\hline Knife & & & & & & 1 & \\
\hline Blunt & 1 & & & & & & \\
\hline Fall & & & & & & & 1 \\
\hline
\end{tabular}

interposition because the surgeon considered that, as it was an adolescent, it should be given a hypothesis of not losing one of the limb major vessels. Ligations were performed when adequate perfusion of the limb was evident without reconstruction due to the presence of collaterals. These included ligation of 2 radial arteries, 4 ulnar arteries, 1 common palmar digital artery, and 1 anterior tibial artery. Adjunct procedures included the following: one 4-compartment leg fasciotomy due to associated soft tissue trauma, 8 tendon repairs, and 11 nerve repairs (Table III). Operations for venous injuries included 4 ligations and 2 primary repairs (Table IV). There were no deaths or major complications; following surgery, all patients were prescribed postoperative antibiotics as recommended for contaminated wounds, and no major infection was present postoperatively. There was no limb loss in this series.

The median length of stay in the hospital was 6 days (range: $2-23$ days). Six patients spent at least 1 day in the pediatric intensive care unit. After operation, anticoagulation (enoxaparin $\mathrm{l} \mathrm{mg} / \mathrm{kg}$ per dosage, twice a day) was considered for patients considered at high risk of occlusion or when venous trauma was associated due to the risk of venous thrombosis. This was considered necessary in 5 patients, and anticoagulation was started only after 4-6 hr and only when there was no evidence of bleeding, and continued for at least 7 days. The choice of low-molecular-weight heparin is controversial due to the incomplete reversal of anti-Xa activity in case of bleeding. However, there were no cases of bleeding and no patients required discontinuation or reversal of heparin. These were 4 patients with arterial injuries and vein grafts interpositions and the patient with ulnar artery injury submitted to end-to-end anastomosis. The patient with reconstruction of posterior tibial artery with vein graft interposition was kept on acetylsalicylic acid for 2 months.

The median time of follow-up was 52 months (range: 20-94 months). At the most recent appointment, 10 patients were asymptomatic and 11 patients had impaired mobility and/or decreased sensation, but no patient was severely disabled and all could perform normal daily living activities. There were no limb asymmetries or dysfunction in patients with arterial injuries that were ligated.

\section{DISCUSSION}

Pediatric noniatrogenic vascular injuries are rare situations that may pose significant therapeutic 
Table III. Surgical procedures in arterial injuries

\begin{tabular}{|c|c|c|c|c|c|c|}
\hline \multirow[b]{2}{*}{ Artery injured } & \multicolumn{6}{|c|}{ Surgical procedures } \\
\hline & Primary repair & Vein graft repair & Ligation & Fasciotomy & Tendon repair & Nerve repair \\
\hline Brachial & & 1 & & & & \\
\hline Radial & 1 & & 2 & & 1 & 1 \\
\hline Ulnar & 3 & & 4 & & 4 & 7 \\
\hline Proper palmar digital & 1 & & & & 1 & 1 \\
\hline Common palmar digital & & & 1 & & 1 & 1 \\
\hline Superficial Femoral & & 2 & & & & \\
\hline Anterior tibial & & & 1 & 1 & & \\
\hline Posterior tibial & & 1 & & & 1 & 1 \\
\hline Total & 5 & 4 & 8 & 1 & 8 & 11 \\
\hline
\end{tabular}

Table IV. Surgical procedures in venous injuries

\begin{tabular}{lll}
\hline & \multicolumn{2}{l}{ Surgical procedures } \\
\cline { 2 - 3 } Vein injured & Primary repair & Ligation \\
\hline External jugular & & 1 \\
$\begin{array}{lll}\text { Collaterals of internal jugular } \\
\text { Femoral }\end{array}$ & 1 & 1 \\
$\begin{array}{l}\text { Anterior tibial } \\
\text { Posterior tibial }\end{array}$ & 1 & 1 \\
Right suprahepatic & & \\
Total & 2 & 4 \\
\hline
\end{tabular}

challenges. This study examined our outcomes managing children with traumatic vascular injuries, demonstrated excellent limb preservation and adequate recovery irrespective of the procedure. Over the course of follow-up, all reconstructions remained patent; ligation, when chosen, resulted in no consequence in terms of function or growth. Also, we did not have any operative mortality (same as Kirkilas et al.; Meagher et al., however, mentioned a 13\% operative mortality, mainly because of intraoperative exsanguinating hemorrhage).

Penetrating trauma by glass was the most common injury mechanism as it is reported in literature. $^{1,4,5}$ Also in accordance to literature, the upper and lower limbs were the most commonly injured locations. ${ }^{1,2}$ This explains the relatively high number of patients managed by arterial ligation-all cases included were either arm or leg injuries, where at least one other major artery provided adequate tissue perfusion, and no signs of ischemia were present.

In this series, all documented vascular injuries were managed operatively, as did Kirkilas et al. and Meagher et al. All patients were taken to the operating theatre for exploration of the injury under general anesthesia because of the inability to tolerate exploration of the injury secondary to patient collaboration. Primary anastomosis was attempted whenever it was possible. When primary repair was not feasible, autologous venous grafts were used, as they have demonstrated the best results. ${ }^{4-7}$ Reversed great saphenous vein was always used, as it is consensually the preferred autologous conduit; synthetic conduits, such as polytetrafluoroethylene or Dacron, were never used as they are more prone to infection and higher thrombosis rates with poor long-term patency, especially in children. ${ }^{1,5}$ Because of consideration for growth, interrupted sutures repairs are frequently used. ${ }^{1,4-6}$ Interrupted suture repair allow for vessel growth that is precluded by circumferential running suture as this causes a "purse stringing" effect with growth. ${ }^{6,8}$ However, generous spatulation of joined conduits in running suture may be an alternative, as it allows for lengthy suture line circumferences that will accommodate later increases in vessel size without stricture. ${ }^{5}$ In our series, we performed mainly interrupted suture repairs with no anastomotic stenosis observed during follow-up.

As in adult vascular reconstruction, we have used interrupted nonabsorbable sutures in these trauma cases without any evidence of subsequent complications. The small caliber of vessels offers less room for error, so great care should be paid to ensure meticulous technique. We also try to minimize the compromise of collaterals; end-to-side anastomoses, in comparison to end-to-end anastomoses, are less likely to interfere with collateral vessels. ${ }^{4}$ All vascular repairs were successful at restoring perfusion.

This study demonstrates a $100 \%$ rate of limb salvage as did Meagher et al.; Corneille et al. had an amputation rate inferior to 3\%; and Kirkilas et al. showed an overall amputation rate of $13 \%$. One fasciotomy was performed (Kirkilas et al. mentioned performance of fasciotomies in $13 \%$ of patients); it was a wound of the superior third of 
the leg made with glass, with section of anterior tibial artery. When fasciotomy is considered necessary in the leg, we routinely do 4-compartment fasciotomy through a medial and lateral incision. The popliteal vessels below the knee are exposed through medial incision as usual, and the deep and superficial posterior compartments are released longitudinally further than required for exposure. No fascia or skin closure is performed. In the case presented, the reason for fasciotomy was the associated soft tissue trauma in a child with large muscle mass resulting in bleeding and edema with consequent neurological deficit and not the presence of ischemia.

All sequelae in follow-up came from associated injuries of adjacent nerves and tendons. Ten patients had short-term complications (within 8 weeks after injury), such as impaired function, which included decreased mobility or range of motion and paresthesia/hyposthesia. Kirkilas et al. found impaired function as the most common complication in short- and long-term follow-up $(60 \%, 75 \%)$.

This retrospective study was limited by its retrospective design, with identification of patients relying exclusively on administrative data. This may have resulted in incomplete capture of all cases over the study period. Also, the study is limited by the relatively small sample of cases. However, although our center is not an official reference trauma center, this series of 21 cases in 7 years $(0.6 \%$ of all pediatric traumatic injuries), 18 of which referred from other hospitals, is similar to worldwide trauma centers in terms of volume. For example, Corneille et al. ${ }^{1}$ at San Antonio, Texas, reported an incidence of $1.4 \%$ significant vascular in- juries over a 13-year period; Kirkilas et al. in Phoenix (USA) describe a cohort of 23 patients over a 6-year period; and Meagher et al. in Houston, Texas (USA) included 50 patients over a 21 -year period.

\section{CONCLUSION}

Noniatrogenic pediatric traumatic vascular injuries are rare. When they do occur, they occur most often in an extremity and are the result of penetrating trauma. Early identification and treatment is critical in order to achieve best functional outcome.

\section{REFERENCES}

1. Corneille M, Gallup T, Villa C, et al. Pediatric vascular injuries: acute management and early outcomes. J Trauma $2011 ; 70: 823-8$.

2. Cox C, Black T, Duke J, et al. Operative treatment of truncal vascular injuries in children and adolescents. J Pediatr Surg 1998;33:462-7.

3. Hammer C, Groner J, Caniano D, et al. Blunt intraabdominal arterial injury in pediatric trauma patients: injury distribution and markers of outcome. J Pediatr Surg 2008;43:916-23.

4. Whitehouse W, Coran A, Stanley J, et al. Pediatric vascular trauma. Arch Surg 1976;111:1269-75.

5. Kirkilas M, Notrica D, Langlais C, et al. Outcomes of arterial vascular extremity trauma in Pediatric trauma. J Pediatr Surg 2016;51:1885-90.

6. Lin P, Barr V, Bush R, et al. Isolated abdominal aortic rupture in a child due to all-terrain vehicle accident. Vasc Endovascular Surg 2003;37:289-92.

7. Linnaus M, Langlais C, Kirkilas M, et al. Outcomes of digital artery revascularization in Pediatric trauma. J Pediatr Surg 2016;51:1543-7.

8. Meagher D, Defore W, Mattox K, et al. Vascular trauma in infants and children. J Trauma 1979;19:532-6. 